\title{
APPLICATION OF A STATISTICAL EMULATOR TO FIRE EMISSION MODELING
}

\author{
Marwan Katurji ${ }^{12^{*}}$, Jovanka Nikolic ${ }^{1}$, Shiyuan Zhong $^{1}$, Scott Pratt ${ }^{3}$, Lejiang Yu ${ }^{1}$, \\ Warren E. Heilman ${ }^{4}$ \\ ${ }^{1}$ Department of Geography, Michigan State University, East Lansing, MI, USA \\ ${ }^{2}$ University of Canterbury Center for Atmospheric Research, Christchurch, New Zealand \\ ${ }^{3}$ Department of Physics and Astronomy, Michigan State University, East Lansing, MI, USA \\ ${ }^{4}$ USDA Forest Service, Northern Research Station, Lansing, MI, USA
}

$\underline{* \text { Corresponding Author's Address }}$

Dr. Marwan Katurji

Center for Atmospheric Research

University of Canterbury

Christchurch, New Zealand

katurji@msu.edu

marwan.katurji@canterbury.ac.nz 


\begin{abstract}
We have demonstrated the use of an advanced Gaussian-Process (GP) emulator to estimate wildland fire emissions over a wide range of fuel and atmospheric conditions. The Fire Emission Production Simulator, or FEPS, is used to produce an initial set of emissions data that correspond to some selected values in the domain of the input fuel and atmospheric parameters for the purpose of training the emulator. The emulated emissions are found to be within $\pm 5 \%$ of the FEPS simulated emissions, providing confidence in the potential use of the GP-emulator for this and other similar applications. Cluster analysis for 1000 emulator-produced posterior samples spanning a wide-range of fuel and environmental conditions suggest that the emulator not only produces valid results but also preserves the physical relationships between the fire emission and the fuel and environmental conditions. Results show that the GP-emulator could be used as an alternative to the simulations from the FEPS modeling system when four or more input parameters related to fuel type, fuel moisture, and weather condition are allowed to vary. This work also provides a conceptual basis for constructing a nation-wide emissions inventory based on a trained GP-emulator representing the complex geographic distribution of fuel types and environmental conditions.
\end{abstract}

Keywords: Fire emission; Statistical Emulator; Simulator; FEPS; emission prediction; Gaussian processes. 


\section{INTRODUCTION}

A Gaussian-Process (GP) emulator is a Bayesian-based statistical tool that treats a set of input and output parameters as indices to stochastic Gaussian functions with a set of mean and covariance values emerging from the input/output parameters. Examples of other algorithms that approach the problem of interpolation or prediction in a nonBayesian framework could be linear/non-linear regression models where a significant assumption of the relationship between the input/output is made, or data-demanding artificial neural network algorithms. Rasmussen (1996) compared up to 7 different methods with large input/output datasets and concluded that the Bayesian approach coupled with a Gaussian process outperforms other traditional methods. For a complete description of how a GP-emulator could be constructed, refer to Chiles and Delfiner (1999), Cressi (1993), Rasmussen and Williams (2006). Several recent studies have applied GP-emulator to complex geophysical processes. Tokmakian and Challenor (2014) and Tokmakian et al. (2012) have shown how GP-emulators could be used to gain specific understanding of complex geophysical systems and interactions within these systems. They also demonstrated how emulators, when trained with data from atmospheric general circulation models (AGCM) and observations, become a powerful tool to assess uncertainties related to the non-linearity in ocean-atmosphere interactions. Holden et al. (2013) demonstrated how an emulator could be used to give spatially and temporally downscaled climate projections, while Gomez et al. (2012), for an extraterrestrial application, showed how the emulation of historic galactic formation could help with the analyses of the current observations of Milky Way-like galaxies. The advantages of fast statistical models lie in the ability of producing large data sets emulating the simulator while performing at a fraction of the computational cost. An example of an application we have chosen here is for fire emissions modeling.

Wildland fires, whether natural or human-ignited, have one thing in common, and that is the release of oxocarbons, hydrocarbons, and particulate matter. Fire smoke can have profound environmental impacts that range from degradation of visibility (Shelby and Speaker 1990; Toman et al. 2004), production of regional haze and smog (van der Werf et al. 2010; Phuleria et al. 2005) and alteration of ecosystem respiration and production 
(Amiro et al. 2010). Smoke from large fires can affect local, regional, and global climate by modifying the earth's radiative balance and altering cloud and precipitation patterns (Oris 2013; Liu et al. 2014). In addition to its impact on natural environments, fire smoke also poses a direct risk to human health (Dennekamp and Abramson 2011; Johnston et al. 2012). The uncertainty in fire emissions comes not only from the misrepresentation of fuel and environmental conditions, but also from the different methods and assumptions used in fire emission models. Various tools or models have been developed to aid in estimating fire emissions. These models vary in their parameterizations and assumptions and the scale at which they are applied (French et al. 2011). These fire emission models are used to estimate emissions from wildfires and prescribed burns for use in air-quality assessment as well as in atmospheric dispersion models. One of the major uncertainties arising from air pollution dispersion tools is the accuracy of the emission sources at the ground level. The other uncertainty comes from humidity and temperature that play a major role in determining the dispersive characteristics of smoke (Hoadley et al. 2003). Fire emissions are usually specified in numerical and analytical models as a function of the size of the area being burned, fuel characteristics including the amount and type of biomass and its moisture content, a combustion efficiency, an emission factor that relates the burned and emitted mass to the biomass burned (Seiler and Crutzen 1980) and weather conditions such as near surface wind speed and atmospheric stability. Uncertainties in each one of these factors contribute unequally to the overall uncertainty of the estimated emissions. French et al. (2011) compared 5 different widely used models and concluded that despite the 25\% range agreement amongst these models, the largest uncertainty of carbon and particulate emissions comes from the difficulty in quantifying the emissions during the smoldering process, the spatial variability of fuel loading (amount of fuel per unit area) and weather conditions that determine the fuel condition.

Given the large number of factors involved in specifying emissions, a full understanding of the contributions from each factor to the uncertainties of estimated emissions is difficult because it requires a large number of tests and model runs. Similarly, a complete understanding of the sensitivity of the total emissions to individual factors and 
interactions of multiple factors also poses a significant challenge. This challenge can be met by the use of statistical tools. In this study, we demonstrate the potential use of a GPemulator constructed for the Models and Data Analysis Initiative (MADAI: $\underline{w w w . m a d a i-}$ public.cs.unc.edu) (O’Hagan 2006; Oakley and O'Hagan 2004) as an alternative to a large number of simulations using a simulator, which in this case is the fire emission model.

What we will show in this short contribution is a new area of applications of a statistical emulator: the area of fire emissions modeling. Fire emission models usually involve a large number of input parameters and it is difficult to fully understand how the interactions among the input parameters affect the emissions output. We will show that the emulator, after being trained by output data of simulated emissions and their corresponding input parameters, can be used to easily expand the emissions database to cover a wide variety of the fuel and atmospheric conditions, thus enabling a better understanding of the dependency of fire emissions to variations of the input parameters and their interactions. In addition to improving the current understanding, the outcome of this study will provide an alternative way of specifying the emissions during a fire event without having to actually run an emissions model, because the emulator itself has been "trained" by the emissions model. The proven success of the GP-emulator to emulate the FEPS model could also suggest a conceptual framework that would allow for constructing a geographic information system or GIS-based nation-wide emission inventory where fuel models (defined here by the fuel type and environmental conditions) are very complex in their geographic distribution and type.

\section{METHODOLOGY}

In this study, we aim to achieve a successful emulation of wildland fire emissions. We will use a specific fire emissions dynamic simulator to produce a small dataset of input parameters and emissions output values that will be used to train an emulator to produce the desired emission products. The results from the emulator will then be validated with a 
new set of simulated results based on the emulator's posterior, or newly produced, samples.

\subsection{The simulator}

The numerical simulator used in this study for dynamically relating fire emissions to the control input parameters is the Fire Emission Production Simulator (FEPS) (Anderson et al. 2004), which is used by many fire and forest managers in the U.S. to estimate fuel consumption and fire emissions and has been successfully integrated into smoke modeling frameworks such as the BlueSky framework (Larkin et al. 2009). Through a graphically driven user interface, FEPS allows users to customize all input variables (such as fuel loading, fuel moisture, consumption, fire growth, hourly meteorological data, etc) for their specific needs and locations in order to calculate emissions for a wide range of fuel and environmental conditions. For fuel loading, the user has the flexibility to choose from 5 natural fuel loading profiles (canopy, shrub, grass, woody, litter), 2 slash fuels (broadcast and piles) and a duff layer as an additional fuel profile, or choose from 24 predefined National Fire Danger Rating System (NFDRS) fuel models (Bradshaw et al 1984). FEPS also allows the user to enter a time varying meteorological field from surface weather stations or numerical weather forecasts, including wind speed at transport height, wind speed at the flame level, and an atmospheric stability class of the ambient atmosphere. For fuel moisture, the user can assign one of six moisture profiles for every fuel type. The output of FEPS includes an emission rate report that contains carbon monoxide $(\mathrm{CO})$, carbon dioxide $\left(\mathrm{CO}_{2}\right)$, methane $\left(\mathrm{CH}_{4}\right)$, and 2.5-micron particulate matter $\left(\mathrm{PM}_{2.5}\right)$. A complete description of FEPS, including its algorithms and equations, can be found in Anderson et al. (2004).

For this study, FEPS is first used to produce emissions of $\mathrm{CO}, \mathrm{CH}_{4}$ and $\mathrm{PM}_{2.5}$ with three input parameters, namely fuel loading (mass or percent of fuel burned per unit area, $\mathrm{pB}$ ), fuel moisture content (FM), and wind speed (WS), for a fixed fuel type. The same FEPS simulations are repeated for another fuel type, thus, increasing the number of input parameters from three to four (the additional parameter being $\mathrm{pB}$ of the new fuel type). By increasing the number of input parameters (hence increasing the training parameter 
dimensions of the emulator), we aim to investigate whether this process could improve the emulator results. For simplicity, the FEPS simulations assume a fire that lasts for 1 hour and burns 1 acre. The burn areas are additive, i.e., the amount of emissions would double if the areas burned double.

A summary of the simulated FEPS input/output is given in Table 1. The parameters in Table 1 are then used to train the GP-emulator. These parameters were prepared by running FEPS driven by FM, WS, pBf1 and pBf2 as inputs and producing the particulate and gaseous emissions as output. The sensitivity of the emissions of the three output species, $\mathrm{CO}, \mathrm{CH}_{4}$, and $\mathrm{PM}_{2.5}$, to the changes in the input parameters is investigated using the large posterior samples generated by the trained emulator.

Table 1. Summary of the input/output parameters and values used in preparing the emulator training set from FEPS. Blue and green colors correspond to experiment 1 (three-input parameter) and 2 (four-input parameter) respectively. pBf1 and pBf2 correspond to percent burn of fuel type 1 (broadcast) and fuel type 2 (shrub); FM and WS correspond to fuel moisture and wind speed, respectively.

\begin{tabular}{|c|c|c|c|c|}
\hline & FM & WS $\left(\mathrm{ms}^{-1}\right)$ & pBf1 (\%) & pBf2 (\%) \\
\hline $\begin{array}{l}\text { Number of } \\
\text { input } \\
\text { parameter } \\
\text { combinations } \\
\text { to FEPS: } \\
240 \text { for exp1 } \\
432 \text { for exp2 }\end{array}$ & $\begin{array}{l}1 \text { (very dry) } \\
3 \text { (moderate) } \\
6 \text { (very wet) }\end{array}$ & $1,5,10,20$ & $\begin{array}{l}5,20,40,60, \\
80,100 \\
\text { or } \\
5 \text { (step 5) to } \\
100\end{array}$ & $\begin{array}{l}5,20,40,60, \\
80,100\end{array}$ \\
\hline $\begin{array}{l}\text { Output from } \\
\text { FEPS }\end{array}$ & $\mathrm{PM}_{2.5}\left(\mathrm{gs}^{-1}\right)$ & CO $\left(g s^{-1}\right)$ & $\mathrm{CH}_{4}\left(\mathrm{gs}^{-1}\right)$ & \\
\hline $\begin{array}{l}\text { Max. } \\
(\exp 1, \exp 2)\end{array}$ & 154,203 & 1905,2506 & 90,118 & \\
\hline $\begin{array}{l}\text { Mean } \\
(\exp 1, \exp 2)\end{array}$ & 50,77 & 609,936 & 29,44 & \\
\hline $\begin{array}{l}\text { Std. Deviation } \\
\text { (exp1, exp2) }\end{array}$ & 37,44 & 455,539 & 21,25 & \\
\hline
\end{tabular}




\subsection{The emulator}

We used a GP-emulator trained by simulated inputs/outputs from FEPS. The GPemulator used in this study is part of the open source statistical packages developed by the Models and Data Initiative program (MADAI: www.madai-public.cs.unc.edu). The statistical package tools and thorough documentation can be downloaded from here (https://madai-public.cs.unc.edu/statistical-tools/distribution-sampling-library). The trained GP-emulator can then be used as a surrogate to FEPS simulations and generate posterior samples of which selected samples can be validated against equivalent FEPS simulations.

The emulator is first trained by the FEPS output for selected input parameter values described in Table 1. Once trained, the GP-emulator is then used to produce 100 posterior samples (input and output parameters). For validation purposes, FEPS is run again with those 100 input parameters to produce a set of output parameters representing 'true' emissions. The outputs in the 100 posterior samples representing the emulated emissions are compared against the 'true' emissions. Figure 1 summarizes the necessary steps involved in the emulation of the FEPS modeling system. 


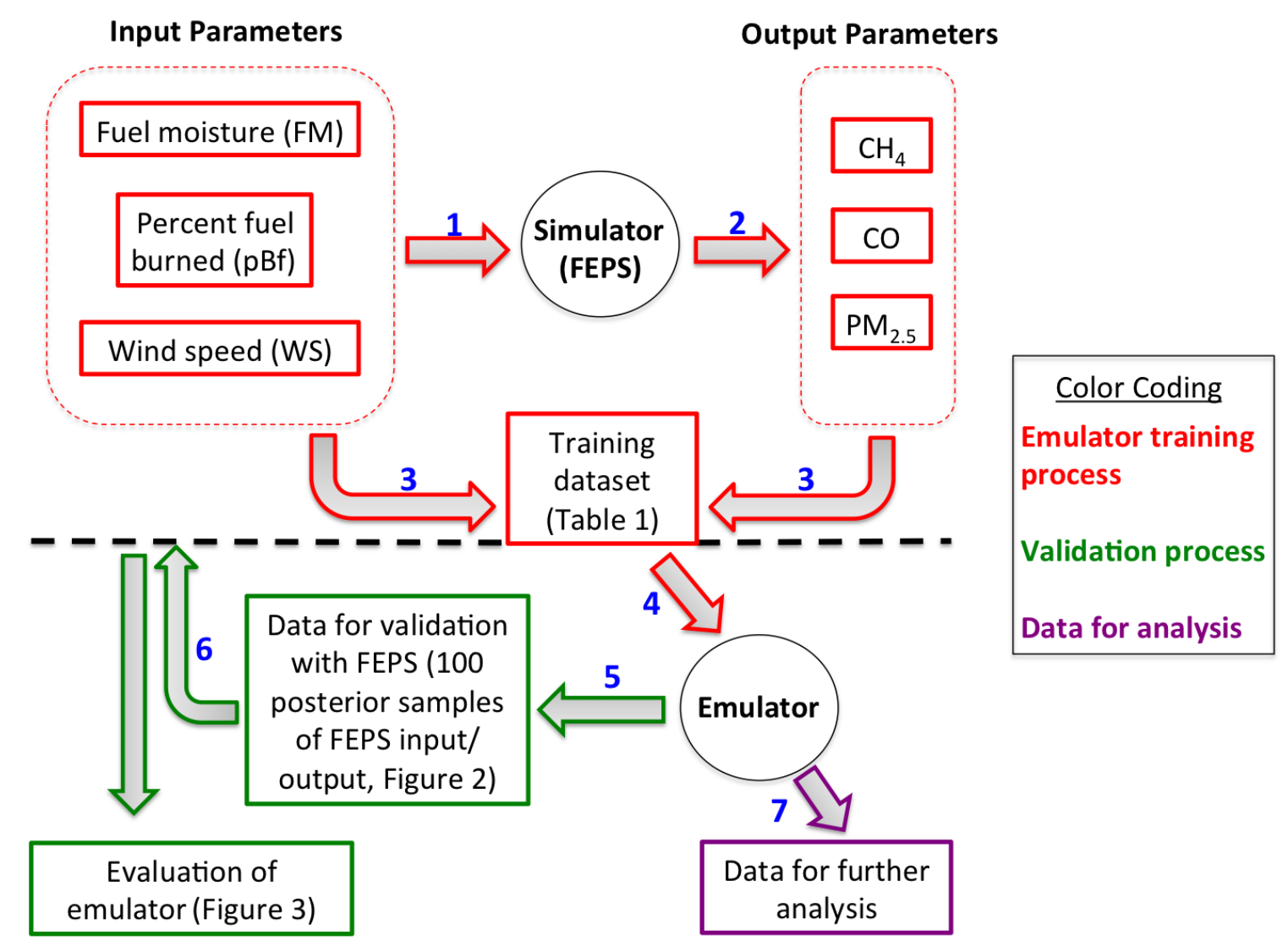

Figure 1. Flow chart showing the necessary steps needed for the emulation process. The color coding key shows the three major stages required prior to using data from the emulations for analysis. The blue numbers indicate the process sequence; using the simulator to produce a training dataset and train the emulator (numbers 1,2, 3, and 4); the validation process (number 5) of comparing a subset of posterior samples produced by the emulator with the output from the simulator at the same sampling points (numbers 5 and 6); and finally the usage of the emulator to produce data ready for further analysis (number 7).

A total of 4 sensitivity experiments to GP-emulator tuning parameters are carried out to reach the best combination of control setting parameters that produce the least relative bias between the simulated and the emulated results. We have also advanced systematically from the emulation of one, two, three and four input parameters to check for consistency or errors in the application. 


\section{RESULTS AND DISCUSSION}

Figure 2a shows the emulated 100 sample emissions of the three species. The emissions for the three species vary consistently among the sampling points. Figure $2 b$ shows three examples of selected points used to train the GP-emulator (the vertical straight lines) and the 1000 posterior samples produced by the trained GP-emulator. The posterior samples fill in the space amongst the training points and are shown to have a matching magnitude distribution depicted by the color intensity of one of the output emissions (CO). These results reveal the basic function of the GP-emulator, and that is to correctly interpolate between the training points and provide a large number of new samples that could be used for further analysis.
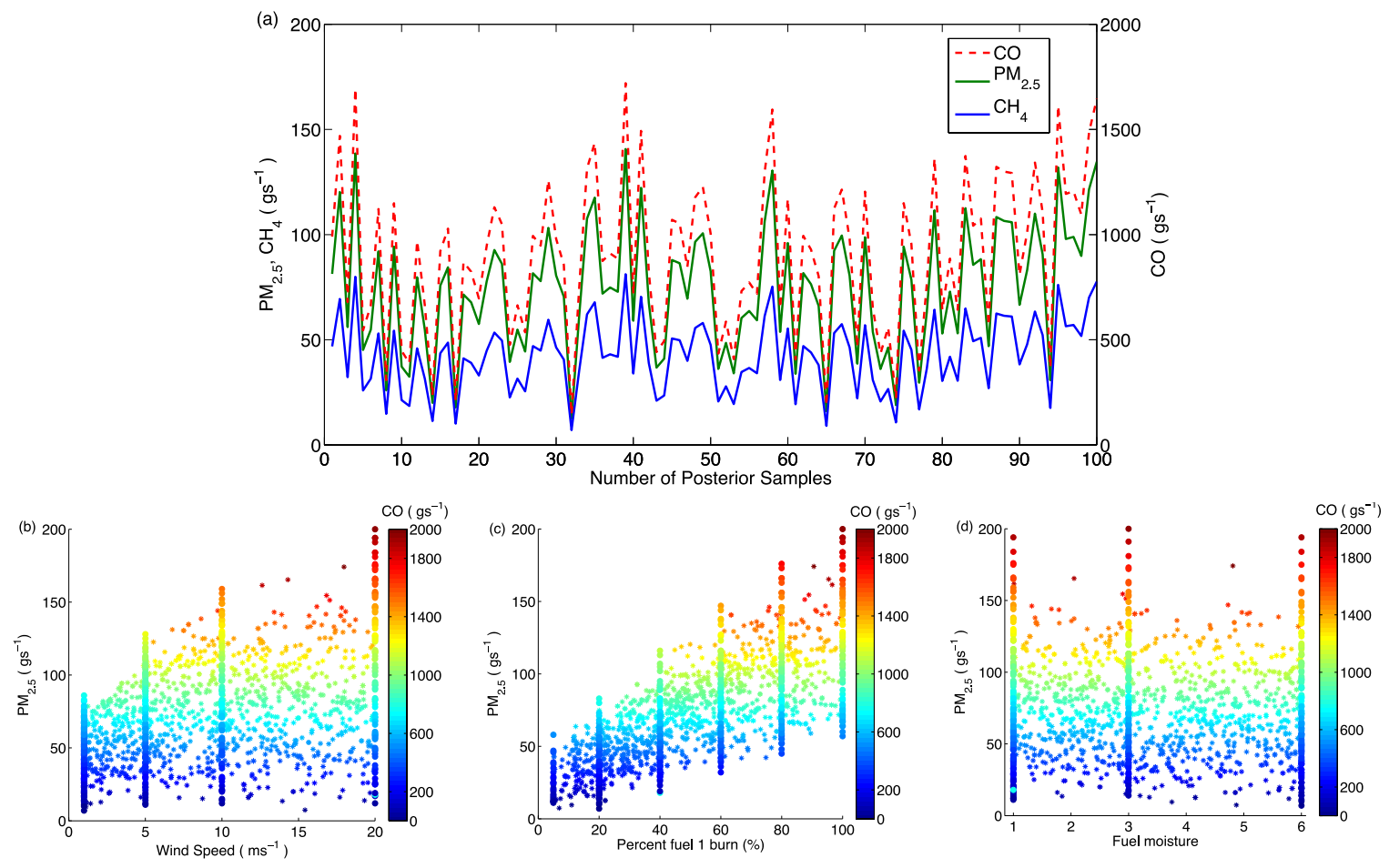

Figure 2. (a) The co-variation of the emulated 100 posterior outputs, (b to d) the filled parameter space (star symbol, total of 1000 samples) produced by the trained GPemulator with 432 training points (vertical straight-line points). The emissions from $\mathrm{CH} 4$ showed similar results and are not presented in this figure. 
The emulator was developed with variety of applications in mind and users are allowed to select parameters in order to optimize the performance of the emulator for their specific application. For this application, two emulator setup parameters related to the order of the regression model and the type of training algorithm are found to have most influence on the emulation results. For the experiment with three input parameters, the combination of a first order regression model and the exhaustive training algorithm appear to have yielded smaller bias compared to higher order regression and the basic training algorithm (dark blue line of stat 4 case in Figure 3a). Still, the bias is large at $\pm 20 \%$ for almost $90 \%$ of the sampled data (Figure 3b). However, a substantial reduction in the overall bias is achieved by the introduction of the fourth input parameter with at least $90 \%$ of the 100 validation points having a bias within $\pm 5 \%$ (light blue line of stat 4 in Figure $3 \mathrm{~b}$ ). Figure $3 b$ shows the cumulative frequency of the bias where the mean bias of the four-input parameter experiments was $-0.8 \%$ with the standard deviation of $4.4 \%$. Increasing the input parameters from three to four by including a new fuel type (with the parameter pBf2) improved the emulation results substantially regardless of the regression models and training algorithms used.

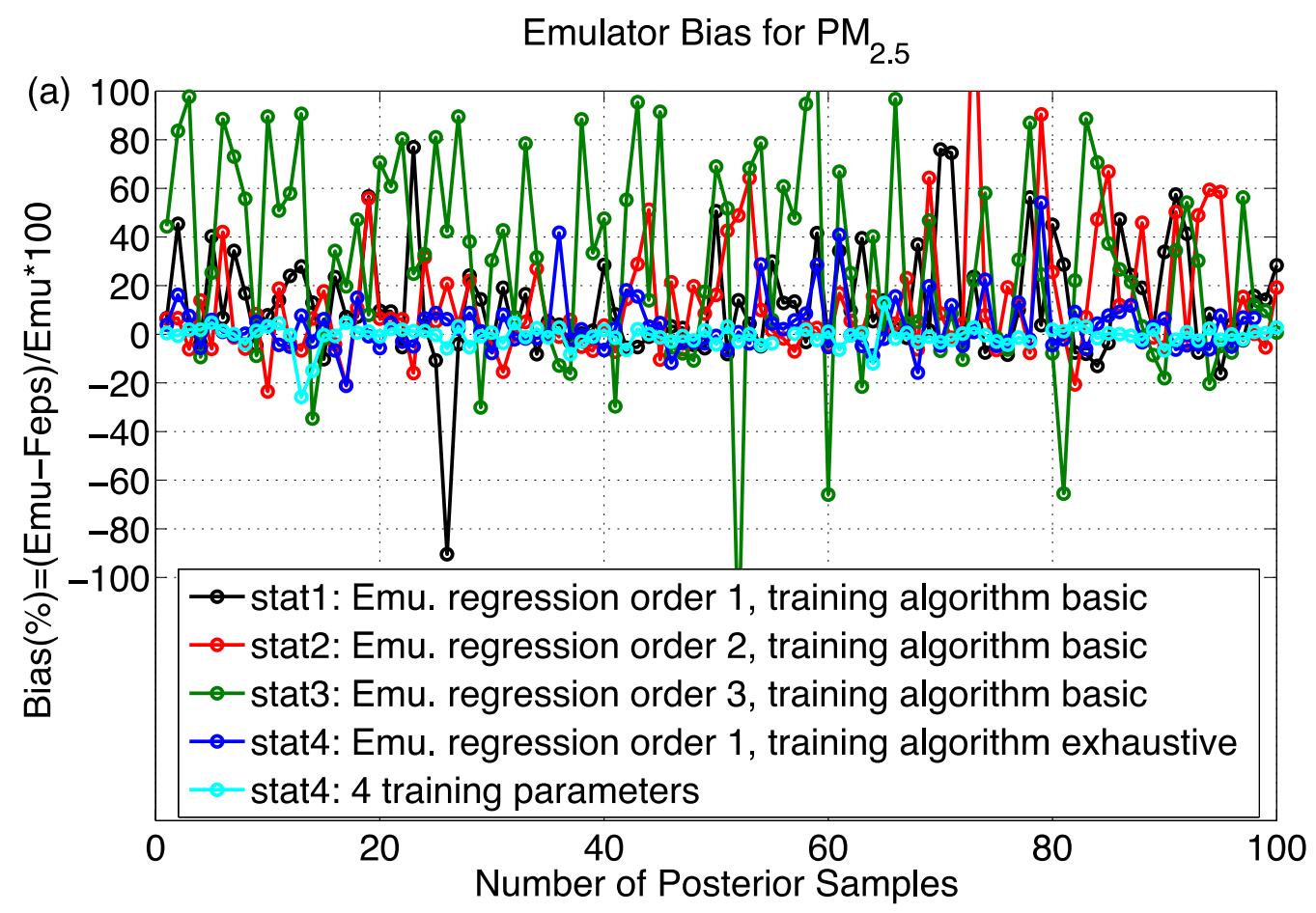




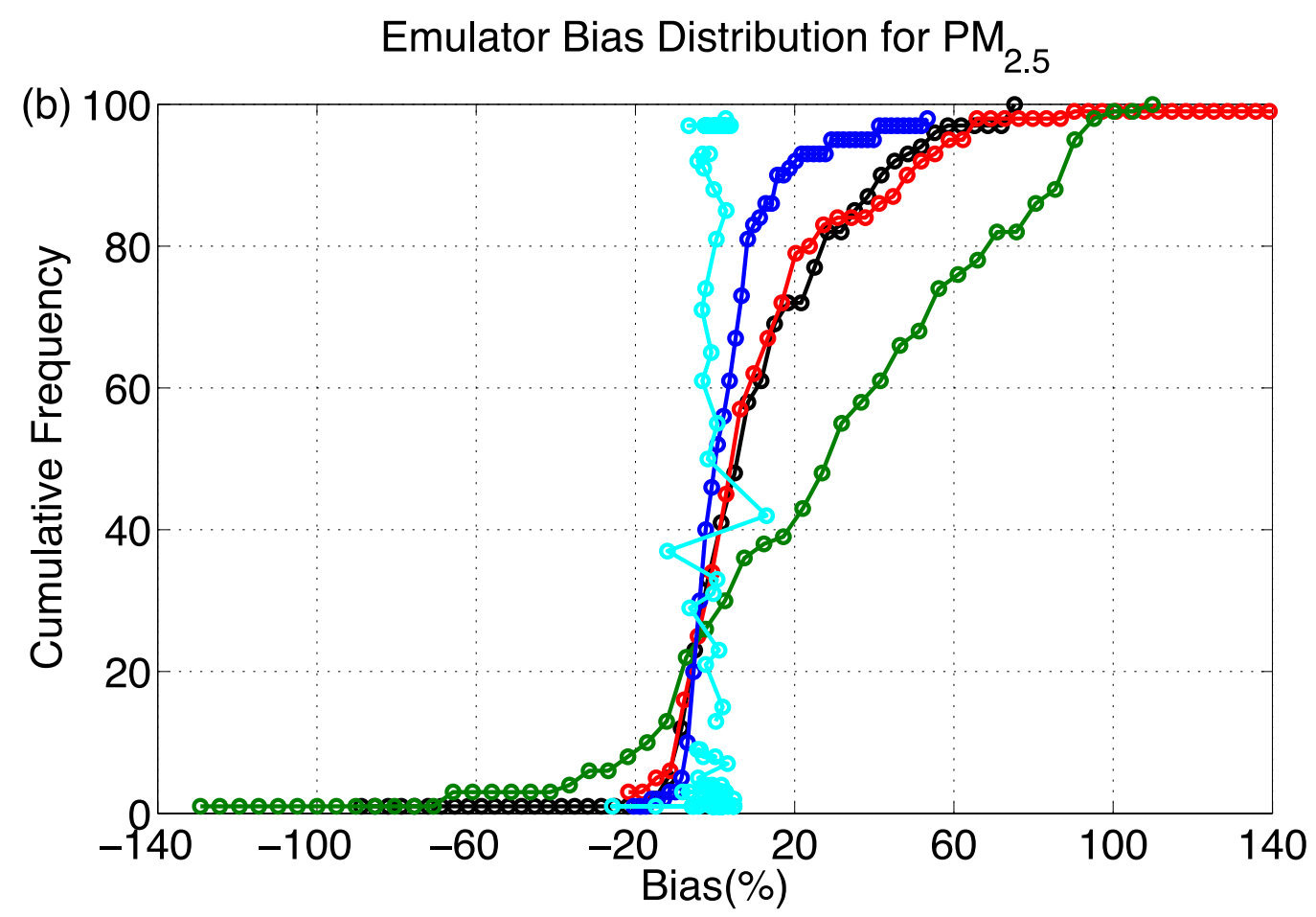

Figure 3. (a) Relative bias (y-axis, (emulator-FEPS/emulator)*100) between the FEPS simulated results for $\mathrm{PM}_{2.5}$ concentrations and the emulated results by the GP-emulator. Four different GP-emulator settings were used (stat1 to 4: see figure inset). (b) Cumulative frequency of the relative bias between the FEPS simulated and GP-emulator emulated results. Both figures have a common legend shown in (a).

To fully explore the sensitivity of the three emission outputs to the four input parameters (pBf1, pBf2, FM and WS), we have used 1000 emulator-produced posterior samples and clustered the output by the k-means algorithm. The algorithm sorts the samples into four different clusters as shown by different colors in Figure 4. The $\mathrm{PM}_{2.5}$ emissions are shown here, but the other two emission outputs produced similar results and only their means are presented in Figure 4. Naturally, the clusters are ordered based on their average values that could be categorized as low, medium-low, medium-high, and high emission scenarios. The mean of each input parameter that corresponds to every cluster is calculated and shown in Figure 4 (inside the color coded boxes). It is to be noted here that these clusters and the corresponding mean values for the four input parameters are a result of the GP-emulator output, not the actual FEPS model output. These results show 
that pBf1 (a part of slash fuel group in FEPS that produces more intense fires and emissions) is correlated with the increase in emissions and has the largest relative change between clusters compared to other input parameters. The other fuel type pBf2 (that comes from the natural fuels category in FEPS and produces less intense fires and emissions) has weak influence on changing the emissions from one cluster to the other. The results also show that the sensitivity of the emission to fuel moisture is weak as indicated by the very little change of mean FM values between the clusters. Wind speeds, on the other hand, are correlated with the increase in emissions, but the impact, judging by the relative change of the mean values between clusters, is not as strong as the input parameter $\mathrm{pBf} 1$. These results, which reveal the true physical dynamics relating the input parameters to the expected fire emissions, add more confidence in the potential use of GP-emulator posterior samples as surrogates to FEPS simulated emissions.

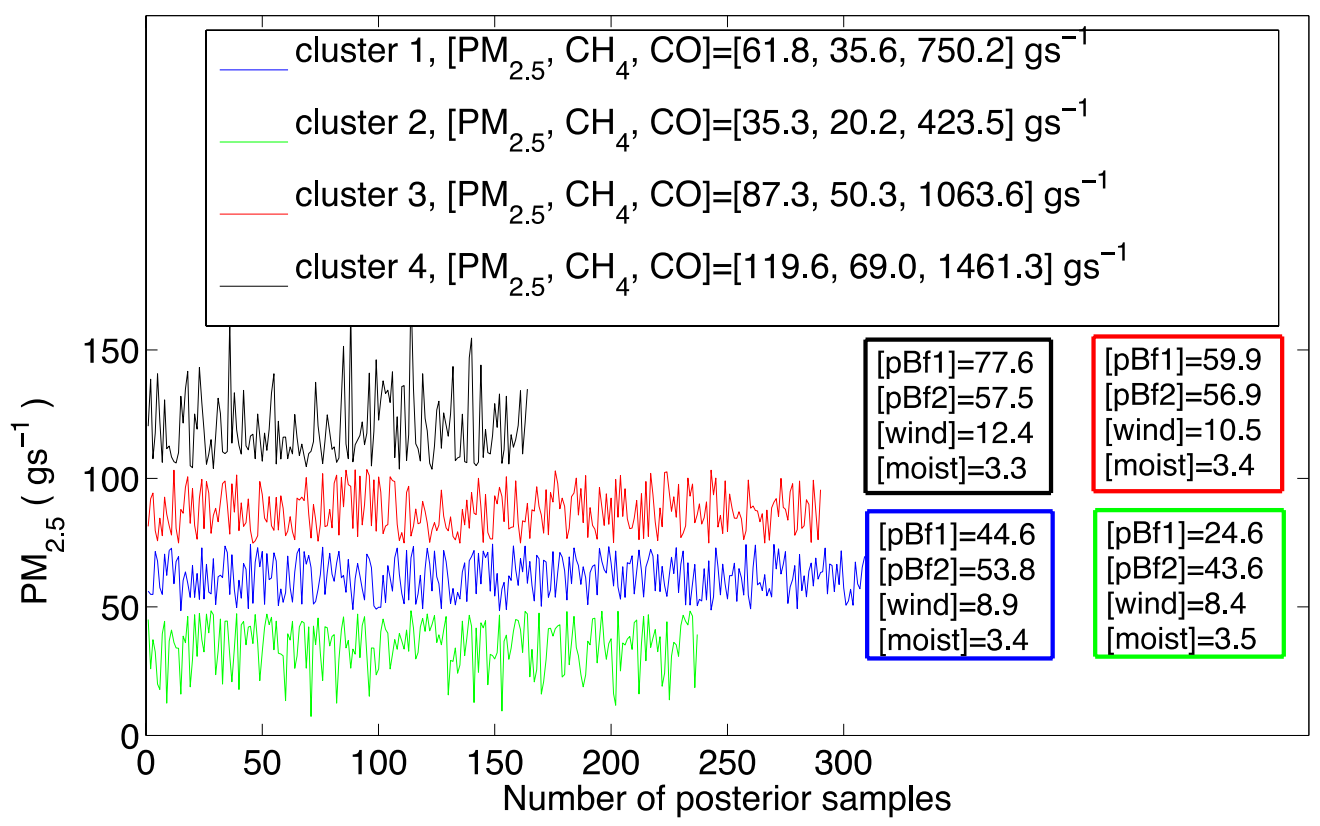

Figure 4. A k-means cluster for 1000 posterior samples (four clusters) and the corresponding mean (in brackets) output emission parameters and the associated mean of the four input parameters of every cluster (inside the color coded boxes).

\section{CONCLUSION}

We have investigated the performance of a GP-emulator driven by a Bayesian framework to emulate the complex physical interactions among several factors contributing to 
wildland fire emissions. The emulated emissions for three species, $\mathrm{CO}, \mathrm{CH}_{4}$ and $\mathrm{PM}_{2.5}$, from wildland fire are within $\pm 20 \%$ of the simulated emissions when three input parameters (fuel loading, fuel moisture and wind speed) are allowed to vary. The relative bias decreased to $\pm 5 \%$ when an additional input parameter, fuel type, is included. Further analysis with 1000 emulator-produced posterior samples representing fire emission and input fuel and environmental properties suggest that the emulator not only produces valid results, but also a large dataset that preserves the relationship between the input and output parameters. An example was provided by relating the maximum emissions to the fuel type known to produce the highest emission rates.

This study represents a pilot-investigation and a proof of concept towards potential use of a GP-emulator to effectively produce fire emissions that span a wide range of fuel and atmospheric conditions. A trained emulator with fast statistical algorithms is capable of producing a very large dataset of valid emission results driven by a smaller set of simulated emissions and the corresponding input parameter values. Although the primary objective of this work is to conceptualize a framework that would allow for constructing a GIS-based nation-wide emission inventory where fuel models (defined here by the fuel type and environmental conditions) are very complex in their geographic distribution and type, one could also allude to various other possible objectives. This work also provides the preliminary results for constructing automated look-up tables relating fire emissions to fuel and environmental properties, which could then be coupled to operational smoke and atmospheric dispersion models driven by weather forecasts or observations. The emulator could also be used to construct an ensemble of fire emission outputs not only driven by one simulator (like FEPS) but with other emissions models. This approach would allow for an uncertainty reduction (due to the incorporation of a collection of fire emission model outputs) and ensemble-based predictions facilitated by a GP emulator.

Although the approach adopted in this study emphasizes the success of the proposed simple statistical methodology in tackling complex environmental physical processes, a continuous case-by-case validation process is necessary. We also recommend that the number and size of training parameters be as large as possible (limited by practicability). 
In theory, the more feedback processes included in a physical system, the more the need arises to include extra input/output training parameters. Unfortunately, there are no guidelines on the relationships of the number of training parameters required and the complexity of the physical process under investigation.

\section{ACKNOWLEDGEMENTS}

This work is supported by Grant PHY-0941373 through the NSF Office of Cyber

Infrastructure. Any opinions, findings, and conclusions or recommendations expressed in this paper are those of authors and do not necessarily reflect the views of the National Science Foundation.

\section{REFERENCES}

Amiro, B. D., et al. 2010. Ecosystem carbon dioxide fluxes after disturbance in forests of North America, $J$. Geophys. Res., 115, G00K02, doi:10.1029/2010JG001390.

Anderson, G. K., Sandberg, D. V., and Norheim, R A. 2004. Fire emission production simulator (FEPS) user's guide, www.fs.fed.us/pnw/fera/feps/.

Bradshaw, L. S., Deeming, J. E., Burgan, R. E., Cohen, J. D. 1984. The 1978 National Fire-Danger Rating System: technical documentation. Gen. Tech. Rep. INT-169. Ogden, UT: U.S. Department of Agriculture, Forest Service, Intermountain Forest and Range Experiment Station. 44 pp.

Chiles, J.P., and Delfiner, P. 1999. Geostatistics: Modeling Spatial Uncertainty. New York, Wiley.

Cressie, N. A. C. 1993. Statistics for Spatial Data. New York, Wiley.

Dennekamp, M., and Abramson, M. J. 2011. The effects of bushfire smoke on respiratory health, Respirology, 16:198-209.

French, N. H. F., et al. 2011. Model comparisons for estimating carbon emissions from North American wildland fire, J. Geophys. Res., 116, G00K05, doi:10.1029/2010JG001469.

Gómez, F. A., Coleman-Smith, C. E., O’Shea, B. W., Tumlinson, J. , and Wolpert, R. L. 2012. Characterizing the Formation History of Milky Way Like Stellar Halos With Model Emulators, The Astrophysical Journal, 760(2), 112, doi:10.1088/0004-637X/760/2/112.

Holden, P. B., Edwards, N. R., Garthwaite, P. H., Fraedich, K., Lunkeit, F., Kirk, E., Labriet, M., Kanudia, A., and Babonneau, F. 2013. PLASIM-ENTSem : A spatio-temporal emulator of Ocean future climate change for impacts assessment, Geoscientific Model Development Discussions, 6, 33493380, doi:10.5194/gmdd-6-3349-2013. 
Hoadley, J., Rorig, M., Westrick, K., Bradshaw, L., Ferguson, S., Goodrick, S., and Werth, P. 2003. Assessing the value of increased model resolution in forecasting fire danger. 5th Symposium on Fire and Forest Meteorology, American Meteorology Society, November 2003, Orlando, Florida.

Johnston, F. H., et al. 2012. Estimated global mortality attributable to smoke from landscape fires, Environmetal Health Perspect, 120: 695-701.

Larkin, N. K., O’Neill, S. M., Solomon, R. S., Raffuse, S., Strand, T., Sullivan, D. C., Krull, C., Rorig, M., Perterson, J. L., and Ferguson, S. 2009, The BlueSky smoke modeling framework. Int. J. Wildland Fire, 20, 18:906-920.

Liu, Y, Goodrick, S., and Heilman, W. 2014. Wildland fire emissions, carbon, and climate: Wildfireclimate interactions. Forest Ecology and Management, 317:80-96.

Oakley, J. E., O'Hagan, A. 2004. Probabilistic sensitivity analysis of complex models: a Bayesian approach, Journal of the Royal Statistical Society: Series B (Statistical Methodology), 66, 751-769, doi: 10.1111/j.1467-9868.2004.05304.x.

O'Hagan, A. 2006. Bayesian analysis of cimputer code outputs: A tutorial, Reliability Engineering and System Safety, 91, 1290-1300, doi:10.1016/j.ress.2005.11.025

Oris, F., Asselin, H., Ali, A. A., Finsinger, W., Bergeron, Y. 2013. Effect of increased fire activity on global warming in the boreal forest, Environmental Reviews, 22(3): 206-219, doi: 10.1139/er-20130062 .

Phuleria, H. C., Fine, P. M., Zhu, Y., and Sioutas, C. 2005, Air quality impacts of the October 2003 southern California wildfires, J. Geophys. Res., 110, D07S20, doi:10.1029/2004JD004626.

Rasmussen, C. E., and Williams, C. K. I. 2006. Gaussian Processes for Machine Learning (Adaptive Computation and Machine Learning), Cambridge, Massachusetts, MIT Press.

Rasmussen, C. E. 1996. Evaluation of Gaussian process and other methods for non-linear regressions. PhD thesis submitted to Department of Computer Science, University of Toronto.

Seiler, W., and Crutzen, P. J. 1980. Estimates of gross and net fluxes of carbon between the biosphere and atmosphere from biomass burning, Clim. Change, 2, 207-247, doi:10.1007/BF00137988.

Shelby, B., and Speaker, R. W. 1990. Public attitudes and perceptions about prescribed burning. In J. D. Walstad, S. R.

Toman, E., Shindler, B., and Reed, M. 2004. Prescribed Fire: The Influence of Site Visits on Citizen Attitudes, The Journal of Environmental Education, 35:3, 13-33, doi: 10.3200/JOEE.35.3.13-33.

Tokmakian, R., and Challenor, P. 2014. Uncertainty in modeled upper ocean heat content change, (February 2013), Climate Dynamics, 823-842, doi:10.1007/s00382-013-1709-9.

Tokmakian, R., Challenor, P., and Andrianakis, Y. 2012. On the Use of Emulators with Extreme and Highly Nonlinear Geophysical Simulators, Journal of Atmospheric and Oceanic Technology, 29, 1704-1716, doi:10.1175/JTECH-D-11-00110.1.

van der Werf, G. R., Randerson, J. T., Giglio, L., Collatz, G. J., Mu, M., Kasibhatla, P. S., Morton, D. C., De Fries, R. S., Jin, Y., and van Leeuwen, T. T. 2010. Global fire emissions and the contribution of 
deforestation, savanna, forest, agricultural, and peat fires (1997-2009), Atmos. Chem. Phys. Discuss., 10, 16,153-16,230, doi:10.5194/acpd-10-16153-2010. 